Pacific Journal of Mathematics

HARMONIC ANALYSIS OF SPHERICAL FUNCTIONS ON 


\title{
HARMONIC ANALYSIS OF SPHERICAL FUNCTIONS ON REDUCTIVE GROUPS OVER $p$-ADIC FIELDS
}

\author{
M. TADIĆ
}

\begin{abstract}
We introduce spaces $\mathcal{C}^{\gamma}(G, K), 0<\gamma \leq 2$, of rapidly decreasing $K$-bi-invariant functions on a reductive $\mathfrak{p}$-adic group $G$ where $K$ is a maximal compact subgroup of $G$, and we study spherical transformations on these spaces. The image of $\mathcal{C}^{\gamma}(G, K)$ under spherical transformation is completely described when $K$ is a standard maximal compact subgroup of $G$.
\end{abstract}

Introduction. Let $G$ be the group of rational points of a connected reductive algebraic group defined over a locally compact totally disconnected nondiscrete field $k$, with anisotropic center.

The group $G$ is a totally disconnected locally compact group. A maximal compact subgroup $K$ of $G$ is fixed. The set of all (zonal) spherical functions on $G$ with respect to $K$ is parametrized naturally by orbits of the Weyl group $W$ in a commutative complex Lie group $\hat{T}_{\mathbf{C}}$.

For $0<\gamma \leq 2$ a family of Schwartz spaces $\mathrm{C}^{\gamma}(G, K)$ of rapidly decreasing $K$-bi-invariant functions on $G$ is defined. It is shown that they are Fréchet algebras under convolution. For $f \in \mathcal{C}^{\gamma}(G, K)$ its spherical transform is defined by

$$
\hat{f}(s)=\int_{G} f(g) \Gamma_{s}\left(g^{-1}\right) d g
$$

for $s \in \hat{T}$, where $\hat{T}$ is the greatest compact subgroup of $\hat{T}_{\mathbf{C}}$ and $\Gamma_{s}$ is the spherical function corresponding to $s$. $\hat{T}$ is a compact real Lie group.

Let $C_{W}^{\infty}(\hat{T})$ denote the algebra of all $W$-invariant infinitely differentiable functions on $\hat{T}$ under pointwise multiplication. Then the spherical transformation $f \mapsto \hat{f}$ is a continuous epimorphism of $e^{2}(G, K)$ onto $C_{W}^{\infty}(\hat{T})$. With certain additional conditions on $K$, we show that the spherical transformation is an isomorphism of topological algebras. The situation when these additional conditions are fulfilled is called the standard case and the contrary case is called the exceptional [5]. A standard maximal compact subgroup of $G$ may always be found. If $G$ is a split group, these additional conditions are always fulfilled.

If $\gamma<2$, every spherical transform $\hat{f}$ of $f \in \mathcal{C}^{\gamma}(G, K)$ may be uniquely extended to a holomorphic function defined on a $W$-invariant domain $D^{\gamma}$ 
containing $\hat{T}$. If the algebra of all $W$-invariant analytic functions on $D^{\gamma}$ with bounded derivatives is denoted by $\mathcal{H}_{W}\left(D^{\gamma}\right)$, then the spherical transformation is a morphism of topological algebras $\mathcal{C}^{\gamma}(G, K)$ and $\mathcal{H}_{W}\left(D^{\gamma}\right)$. If $K$ is standard, then it is an isomorphism.

In $\S 6$ the spherical transformation of elements of $\bigodot^{2}(G, K)$ with $K$ exceptional is discussed.

We conclude the introduction with a few basic notations.

If $X$ is a locally compact space, then the vector space of all complexvalued continuous functions on $X$ is denoted by $C(X)$ and the subspace of all compactly supported functions is denoted by $C_{0}(X)$. For $G$ a totally disconnected locally compact unimodular group and $K$ its open compact subgroup, the vector space of all $K$-bi-invariant functions on $G$ is denoted by $C(G, K)$. Let $C_{0}(G, K)=C_{0}(G) \cap C(G, K)$. The space of all $\gamma$-integrable functions in $C(G, K)$ is denoted by $L^{\gamma}(G, K), 0<\gamma \leq 2$. We regard $L^{\gamma}(G, K)$ as a topological vector space in a standard way.

$\mathbf{Z}$ will denote the ring of rational integers, $\mathbf{R}$ and $\mathbf{C}$ will denote the fields of real and complex numbers, respectively. The set of all non-negative rational integers is denoted by $\mathbf{Z}_{+} \cdot \sqrt{-1}$ is denoted by $i$.

The present paper is an account of the results of the author's thesis. It is a pleasure to acknowledge the help given to me by my advisor, Professor D. Miličić. The results presented in this paper are analogous to the results of P. C. Trombi and V. S. Varadarajan for real semisimple Lie groups.

1. Spherical functions. Let $k$ be a locally compact totally disconnected non-discrete field. For an algebraic group $\mathbf{X}$ defined over $k, X$ will denote the group of its $k$-rational points.

In this paper a connected reductive algebraic group, whose center is anisotropic, is denoted by $\mathbf{G}$. Following [3], in this section we introduce necessary notations and we present Macdonald's explicit formula for spherical functions on $G$.

Let $\mathbf{G}^{\text {der }}$ be the derived group of $\mathbf{G}, \tilde{\mathbf{G}}$ the simply connected covering of $\mathbf{G}^{\text {der }}$, and $\psi: \tilde{\mathbf{G}} \rightarrow \mathbf{G}^{\text {der }}$ the canonical homomorphism. If $\mathbf{X}$ is a subgroup of $\mathbf{G}, \psi^{-1}(\mathbf{X})$ is denoted by $\tilde{\mathbf{X}}$.

Fix a minimal parabolic subgroup $\mathbf{P}$ of $\mathbf{G}$. Let $\mathbf{A}$ be a maximal split torus contained in $\mathbf{P}, \mathbf{M}$ its centralizer, $\mathbf{U}$ the unipotent radical of $\mathbf{P}, \mathbf{U}^{-}$ the unipotent radical of the parabolic opposite to $\mathbf{P}$.

Let $\Re$ be the Bruhat-Tits building of $\tilde{G}$ [5]. The group $\tilde{G}$ acts on $\Re$. Let $Q$ be the unique apartment in $\Re$ stabilized by $\tilde{A}$. If $\tilde{N}$ is the normalizer of $\tilde{A}$ in $\tilde{G}$, then $\tilde{N}$ stabilizes $Q$. Let $\nu: \tilde{N} \rightarrow \operatorname{Aut}(\mathbb{Q})$ be the corresponding homomorphism. 
There exists a canonical affine root system $\Sigma_{\text {aff }}$ on an affine space $Q$. Let $W_{\text {aff }}$ be the Weyl group of $\Sigma_{\text {aff }}$. We have $\operatorname{Im} \nu=W_{\text {aff }}$. With a special point $x_{0} \in \mathbb{Q}$ fixed, we will consider only the vector space structure with the origin $x_{0}$ on $Q$. Set

$$
\Sigma=\left\{a \in \Sigma_{\mathrm{aff}} ; a(0)=0\right\}, \quad W=\left\{w \in W_{\mathrm{aff}} ; w(0)=0\right\} ;
$$

$\Sigma$ is a reduced root system and $W$ is its Weyl group. With a $W$-invariant scalar product $($,$) on \mathbb{Q}$ fixed, a canonical isomorphism $\Lambda: \mathbb{Q} \rightarrow \mathbb{Q}^{*}$ is fixed, so we also have the scalar product on $Q^{*}$, the space of linear forms on $Q$.

For $a \in \Sigma_{\text {aff }}$ let $\tilde{U}(a)$ be the group of all $u \in \tilde{U} \cup \tilde{U}^{-}$such that $u x=x$ for all $x \in \mathbb{Q}$ with $a(x) \geq 0$. Let $\Sigma^{+}$be the set of all $\alpha \in \Sigma$ such that $\tilde{U}(\alpha) \subseteq \tilde{U}$. $\Sigma^{+}$is a system of positive roots in $\Sigma$. Let $\Delta$ be the set of simple roots in $\Sigma^{+}$. The rank of $\Sigma$ is denoted by $l$. Set $r_{0}=$ card $\Sigma^{+}$.

Set

$$
\begin{aligned}
& C_{0}=\left\{x \in \mathbb{Q} ; \alpha(x)>0 \text { for all } \alpha \in \Sigma^{+}\right\}, \\
& C_{0}^{\perp}=\left\{x \in \mathbb{Q} ;(y, x) \geq 0 \text { for all } y \in C_{0}\right\} .
\end{aligned}
$$

The closure of $C_{0}$ is denoted by $\bar{C}_{0}$. Let $C$ be the affine chamber of $\mathbb{Q}$ contained in $C_{0}$ having 0 as vertex. Let $\tilde{B}$ be the Iwahori subgroup fixing the chamber $C$. For $w \in W_{\text {aff }}, q(w)$ denotes $[\tilde{B} w \tilde{B}: \tilde{B}]$.

If $x \in \mathbb{Q}, x \neq 0$, we write $x=2 \Lambda^{-1} x /(x, x)$. For $x \in \mathbb{Q}, \tau(x)$ denotes the translation of $\mathbb{Q}$ by vector $x$. If $a \in \Sigma_{\text {aff }}$, the orthogonal reflection of $\mathbb{E}$ with respect to the hyperplane $\{x \in \mathbb{Q} ; a(x)=0\}$ is denoted by $w_{a}$. For $\alpha \in \Sigma$ set $a_{\alpha}=w_{\alpha} w_{\alpha-1}$. Then $a_{\alpha}=\tau(-\alpha)$.

Set $q_{a}=[\tilde{U}(a-1): \tilde{U}(a)]$ for $a \in \Sigma_{\text {aff }}$. Then $q_{a+2}$ is always the same as $q_{a}$. For $\alpha \in \Sigma$ we set $q_{\alpha / 2}=q_{\alpha+1} q_{\alpha}^{-1}$. Let

$$
\Sigma_{1}=\left\{\alpha \in\left(\Sigma \cup \frac{1}{2} \Sigma\right) ; q_{\alpha} \neq 1\right\} .
$$

Then $\Sigma_{1}$ is a root system.

The group $G$ acts on $\tilde{G}$ and $B$. If the normalizer of $A$ in $G$ is denoted by $N$, then $\mathbb{Q}$ is stabilized by $N$. Let $\nu: N \rightarrow \operatorname{Aut}(\mathbb{Q})$ be the corresponding homomorphism. The inverse image of the set of translations is $M$. Set $T=\nu(M)$. The kernel of $\nu$ is denoted by $M_{0}$. We identify $M / M_{0}$ and $T$.

The groups $N / M, \tilde{N} / \tilde{M}$, and $W$ are isomorphic. The Weyl group $W$ acts on $\tilde{M}$ and $M$ by inner automorphisms and $W$ acts on $T$ as well.

Let

$$
B=\{g \in G ; g x=x \text { for all } x \in C\}, \quad K=\{g \in G ; g 0=0\} .
$$

The groups $B$ and $K$ are open compact subgroups and $K$ is a special good maximal compact subgroup of $G[\mathbf{1}]$. 
The case when $q_{\alpha / 2} \geq 1$ for all $\alpha \in \Sigma$ will be called standard. The situation when $q_{\alpha / 2}<1$ for some $\alpha \in \Sigma$ is called the exceptional case [5]. We will also say $K$ is a standard maximal compact subgroup of $G$ or $K$ is exceptional. There is always a special point $x_{0} \in \mathbb{Q}$ such that $K$ is standard.

Set

$$
\begin{aligned}
& M^{-}=\left\{m \in M ;-\nu(m)(0) \in \bar{C}_{0}\right\}, \\
& M_{-}=\left\{m \in M ;-\nu(m)(0) \in C_{0}^{\perp}\right\}, \\
& T^{-}=\nu\left(M^{-}\right), \quad T_{-}=\nu\left(M_{-}\right) .
\end{aligned}
$$

Owing to the Iwasawa decomposition $G=U M K$, the mapping $m \mapsto$ $U m K$ induces a bijection from $M / M_{0}=T$ onto $U \backslash G / K$ ([1] , Proposition (4.4.3)). The composition $G \rightarrow U \backslash G / K \rightarrow T$ is denoted by $h$.

There is the Cartan decomposition $G=K M^{-} K$. The mapping $m \mapsto$ $K m K$ induces a bijection from $T^{-}=M^{-} / M_{0}$ onto $K \backslash G / K$ ([1] , Proposition (4.4.3)). Let $\sigma$ be the composition $G \rightarrow K \backslash G / K \rightarrow T^{-}$.

From the Cartan decomposition we deduce that mapping $f \mapsto f \circ \sigma$ is a bijection from the space of all complex-valued functions on $T^{-}$onto $C(G, K)$. Sometimes we shall identify these two spaces.

If $m_{1}^{-1} \in M^{-}, m_{2} \in M$ and $K m_{1} K \cap U m_{2} K \neq \varnothing$, then $m_{2} m_{1}^{-1} \in M_{-}$, or, equivalently,

$$
\nu\left(m_{1}\right)(0)-\nu\left(m_{2}\right)(0) \in C_{0}^{\perp}
$$

([1], Proposition (4.4.4), (i)).

If $m_{1}, m_{2}, m_{3} \in M^{-}$and $K m_{1} K m_{2} K \cap K m_{3} K \neq \varnothing$, then $m_{1} m_{2} m_{3}^{-1}$ $\in M_{-}$, or, equivalently,

$$
\nu\left(m_{1}\right)(0)+\nu\left(m_{2}\right)(0)-\nu\left(m_{3}\right)(0) \in\left(-C_{0}^{\perp}\right)
$$

([1], Proposition (4.4.4), (iii)).

For $m^{-1} \in M^{-}$we have

$$
K m K \cap U m K=m K
$$

([1], Proposition (4.4.4), (ii)).

Let $\delta$ be the modular character of $P$. It is easy to see that $p \mapsto \delta(\psi(p))$ is the modular character of $\tilde{P}$.

For $\alpha \in \Delta$ choose $n \in \tilde{N}$ such that $\nu(n)=a_{\alpha}$. Then $\delta(n)^{-1}=q_{\alpha / 2} q_{\alpha}^{2}$ ([5], Corollary (3.2.7)). Since $q_{\alpha / 2} q_{\alpha}^{2}=q_{\alpha+1} q_{\alpha}$, it follows that

$$
\delta(n)^{-1}>1 \text {. }
$$


Let $\mu$ be the Haar measure on $G$ such that $\mu(K)=1$. For $g \in G$ we define $v_{g}=\mu(K g K)$. If $t \in T$ and $m \in M$ such that $\nu(m)=t$, then we write $v_{t}=\mu(K m K)$.

The next lemma is proved in [8].

1.1. LEMMA. If $K_{0}$ is an open compact subgroup of $G$, then there exist $c_{1}$, $c_{2}>0$ such that

$$
c_{1} \delta(m)^{-1} \leq \mu\left(K_{0} m K_{0}\right) \leq c_{2} \delta(m)^{-1}
$$

for all $m \in M^{-}$.

A complex character of $M$ is called unramified if it is trivial on $M_{0}$. The mapping $s \mapsto s \circ \nu$ is an isomorphism from the group of all characters of $T$ onto the group of all unramified characters of $M$, so we identify these two groups and denote them by $\hat{T}_{\mathbf{C}}$. The character $\delta \mid M$ is unramified and this character is denoted by $\delta$ as well.

$T$ is a free abelian group of rank $l$. Fix $b_{1}, b_{2}, \ldots, b_{l}$ a basis of $T$. The mapping $\Theta: s \mapsto\left(s\left(b_{1}\right), \ldots, s\left(b_{l}\right)\right)$ is an isomorphism of $\hat{T}_{\mathbf{C}}$ onto $\left(\mathbf{C}^{*}\right)^{l}$. As $\left(\mathbf{C}^{*}\right)^{l}$ is a complex Lie group, there is the unique complex Lie group structure such that $\Theta$ is a complex Lie group isomorphism. This structure on $\hat{T}_{\mathbf{C}}$ does not depend on the choice of basis $b_{1}, \ldots, b_{l}$.

The image of the invariant analytic vector field is ${ }_{k} \partial / \partial s_{k}$ on $\left(\mathbf{C}^{*}\right)^{l}$, $k=1,2, \ldots, l$, under the mapping induced on vector fields by $\Theta^{-1}$, is denoted by $S_{k}$. The set $S=\left\{S_{k} ; k=1, \ldots, l\right\}$ is a basis of the Lie algebra $t$ of $\hat{T}_{\mathbf{C}}$. The universal enveloping algebra of $t$ is denoted by $Q(t)$. Let $\delta$ be the basis of $\mathcal{Q}(t)$ which is obtained from $S$ by the Poincare-BirkhoffWitt Theorem. There is the canonical filtration $\left(\mathcal{Q}_{n}(\mathrm{t}) ; n \in \mathbf{Z}_{+}\right)$of $\mathcal{Q}(\mathrm{t})$. The sets $\delta_{n}=\delta \cap \mathcal{Q}_{n}(\mathrm{t})$ are finite.

Let $\hat{T}$ be the set of all unitary characters in $\hat{T}_{\mathbf{C}}$. Then $\hat{T}$ is a real Lie subgroup of $\hat{T}_{\mathbf{C}}$ and the greatest compact subgroup of $\hat{T}_{\mathbf{C}}$. Clearly, $\Theta(\hat{T})=\left\{z \in \mathbf{C}^{*} ;|z|=1\right\}^{l}$. Denoting by $\left(r_{k}, \varphi_{k}\right)$ the polar coordinates of $z_{k}$, we obtain an invariant vector field $\partial / \partial \varphi_{k}$ on $\Theta(\hat{T})$ and the corresponding vector field $\tilde{S}_{k}$ on $\hat{T}$. The set $\left\{\tilde{S}_{k} ; k=1, \ldots, l\right\}$ is a basis of the Lie algebra $\mathfrak{f}_{0}$ of $\hat{T}$. Let $\tilde{\mathfrak{t}}$ be the complexification of $\tilde{\mathfrak{f}}_{0}$. The elements of $\mathfrak{\mathfrak { f }}$ act on complex-valued smooth functions on $\hat{T}$.

We extend the mapping $\tilde{S}_{k} \mapsto S_{k}, k=1, \ldots, l$, to a Lie algebra isomorphism of $\mathfrak{t}$ onto $\mathfrak{t}$ and to an isomorphism $\omega$ of $\mathcal{U}(\tilde{\mathfrak{t}})$ onto $\mathcal{Q}(\mathfrak{t})$. If an analytic function $\varphi$ is defined on an open set containing $\hat{T}$, then

$$
(\omega X)(x) \varphi=X(x)(\varphi \mid \hat{T})
$$

for $X \in \mathcal{U}(\tilde{\mathfrak{f}})$ and $x \in \hat{T}$. We write $\tilde{\delta}_{n}=\omega^{-1}\left(\delta_{n}\right)$ and $\tilde{\mathscr{S}}=\omega^{-1}\left(\delta_{n}\right)$. 
A character $s \in \hat{T}_{\mathbf{C}}$ is called regular if $s(\tau(\check{b})) \neq 1$ for all $b \in \Sigma_{1}$.

Let $\operatorname{Ind}(s \mid P, G)$ be the admissible representation of $G$ induced by $s \in \hat{T}_{\mathbf{C}}$. It is the right regular representation of $G$ on the space of all locally constant functions $f: G \rightarrow \mathbf{C}$ such that $f(p g)=\left(s \delta^{1 / 2}\right)(p) f(g)$ for all $p \in P, g \in G$. The contragredient of $\operatorname{Ind}(s \mid P, G)$ is isomorphic to $\operatorname{Ind}\left(s^{-1} \mid P, G\right)$, and the canonical form on $\operatorname{Ind}(s \mid P, G) \times \operatorname{Ind}\left(s^{-1} \mid P, G\right)$ is given by

$$
(f, \tilde{f}) \mapsto \int_{K} \tilde{f}(k) f(k) d k
$$

Define $\Phi_{s}: G \rightarrow \mathbf{C}$ by setting $\Phi_{s}(g)=\left(s \delta^{1 / 2}\right)(h(g))$. Then $\Phi_{s}$ is a non-trivial $K$-invariant vector in $\operatorname{Ind}(s \mid P, G)$. Let

$$
\begin{aligned}
\Gamma_{s} & =\int_{K} \Phi_{s}(k g) \Phi_{s^{-1}}(k) d k=\int_{K} \Phi_{s}(k g) d k \\
& =\int_{K}\left(s \delta^{1 / 2}\right)(h(k g)) d k
\end{aligned}
$$

for $g \in G$. Then $\Gamma_{s}$ is the matrix coefficient and zonal spherical function, or, simply, the spherical function corresponding to $s$. Since the contragredient of $\operatorname{Ind}\left(s^{-1} \mid P, G\right)$ is $\operatorname{Ind}(s \mid P, G)$, it is easily seen that

$$
\Gamma_{s}(g)=\Gamma_{s^{-1}}\left(g^{-1}\right) \text { for all } s \in \hat{T}_{\mathbf{C}} \text { and } g \in G .
$$

$W$ acts on $T$; thus $W$ also acts on $\hat{T}_{\mathbf{C}}$ and $\hat{T}$. For $w \in W$ and $s \in \hat{T}_{C}$ we have

$$
\Gamma_{s}=\Gamma_{w s}
$$

([3], Proposition 4.1). The converse will be proved in the next lemma.

1.2. LemMA. If $s_{1}, s_{2} \in \hat{T}_{C}$ and $\Gamma_{s_{1}}=\Gamma_{s_{2}}$, then there exists $w \in W$ such that $s_{1}=w s_{2}$.

Proof. Let us denote by $V$ the subrepresentation of $\operatorname{Ind}\left(s_{1} \mid P, G\right)$ generated by $\Phi_{s_{1}}$. Clearly, there exists a maximal subrepresentation $V_{1}$ of $V$ such that $\Phi_{s_{1}} \notin V_{1}$. By ([2], Corollary 6.3.7), there exists $w_{1} \in W$ such that $V / V_{1}$ is isomorphic to a subrepresentation $V_{2}$ of $\operatorname{Ind}\left(w_{1} s_{1} \mid P, G\right)$ and $V_{2}$ is generated by $\Phi_{w_{1} s_{1}}$. The mapping $f \mapsto \tilde{f}$ from $V_{2}$ into $C^{\infty}(G)$ is defined by

$$
\bar{f}(g)=\int_{K} f(k g) d k
$$


Obviously, $\bar{\Phi}_{w_{1} s_{1}}=\Gamma_{w_{1} s_{1}}$. The mapping $f \mapsto \bar{f}$ is a monomorphism of $G$-representations where $G$ acts on $C^{\infty}(G)$ by right translations. Since $\Gamma_{w_{1} s_{1}}=\Gamma_{s_{1}}$, we have seen that there is a composition factor $V_{2}$ of $\operatorname{Ind}\left(s_{1} \mid P, G\right)$ such that $V_{2}$ is isomorphic to the subrepresentation of $C^{\infty}(G)$ generated by $\Gamma_{s_{1}}$. Thus, $\operatorname{Ind}\left(s_{1} \mid P, G\right)$ and $\operatorname{Ind}\left(s_{2} \mid P, G\right)$ have a common composition factor. By ([2], Theorem 6.3.3) there exists $w \in W$ such that $s_{1}=w s_{2}$.

Relation (1.6) implies $s \mapsto \Gamma_{s}(g)$ is an analytic function on $\hat{T}_{\mathbf{C}}$ for every fixed $g \in G$. Each of these functions is $W$-invariant.

If $s$ is a regular unramified character and $\alpha \in \Sigma$, we introduce

$$
c(\alpha, s)=\frac{\left(1-q_{\alpha / 2}^{-1 / 2} q_{\alpha}^{-1} s\left(a_{\alpha}\right)^{-1}\right)\left(1+q_{\alpha / 2}^{-1 / 2} s\left(a_{\alpha}\right)^{-1}\right)}{1-s\left(a_{\alpha}\right)^{-2}} .
$$

If $\alpha / 2 \notin \Sigma_{1}$ then

$$
c(\alpha, s)=\frac{1-q_{\alpha}^{-1} s\left(a_{\alpha}\right)^{-1}}{1-s\left(a_{\alpha}\right)^{-1}} .
$$

Set

$$
c(s)=\prod_{\alpha \in \Sigma^{+}} c(\alpha, s) .
$$

1.3. Theorem. (Macdonald, [2], Theorem 4.2): Let

$$
Q=\sum_{w \in W} q(w)^{-1}
$$

If $s \in \hat{T}_{\mathbf{C}}$ is regular, then for all $m \in M^{-}$we have

$$
\Gamma_{s}(m)=Q^{-1} \sum_{w \in W} c(w s)\left((w s) \delta^{1 / 2}\right)(m) .
$$

From (1.10) we obtain

$$
\Gamma_{s}(g)=Q^{-1} \sum_{w \in W} c(w s)\left((w s) \delta^{1 / 2}\right)(\sigma(g))
$$

for $g \in G$. Relation (1.7) implies

$$
\Gamma_{s}\left(g^{-1}\right)=Q^{-1} \sum_{w \in W} c\left(w s^{-1}\right)\left(\left(w s^{-1}\right) \delta^{1 / 2}\right)(\sigma(g)) .
$$

The set of all regular characters is an open dense subset of $\hat{T}_{\mathbf{C}}$. Since the functions $s \mapsto \Gamma_{s}(g)$ are analytic on $\hat{T}_{\mathbf{C}}$, we can get $\Gamma_{s}(g)$ as the limit of $\Gamma_{s_{1}}(g)$ with $s_{1}$ regular. In this way we can obtain the formula for $\Gamma_{s}$ for any $s$. In such a way I. G. Macdonald derived the formula for any spherical function ([5], Proposition (4.6.2)). 
Set $\Xi=\Gamma_{1}$. There exists a polynomial function $p$ on $\mathbb{Q}$ such that

$$
\Xi(m)=\delta^{1 / 2}(m) p(\nu(m)(0))
$$

for all $m \in M^{-}$and the degree of $p$ is less than or equal to $r_{0}$ ([5], Proposition (4.6.1)).

For $s \in \hat{T}, \Gamma_{s}$ is a positive definite spherical function. Note that every positive definite spherical function $f$ on $G$ satisfies

$$
\begin{gathered}
\overline{f(g)}=f\left(g^{-1}\right), \\
|f(g)| \leq f(1)
\end{gathered}
$$

for all $g \in G$.

2. The algebras $e^{\gamma}\left(G, K_{0}\right)$. Our first aim is to describe a connection between the asymptotic behaviour of $\Xi$ and $\delta$. Let \|\| be the norm on $\mathbb{Q}$ corresponding to the fixed scalar product on $\mathbb{Q}$.

2.1. Proposition. There exist $c_{1}, c_{2}>0$ such that

$$
c_{1} \delta^{1 / 2}(\sigma(g)) \leq \Xi(g) \leq c_{2}(1+\|\sigma(g)(0)\|)^{r_{0}} \delta^{1 / 2}(\sigma(g))
$$

for all $g \in G$.

Proof. The right inequality of (2.1) is an immediate consequence of (1.13).

For $g \in G$ choose $m \in M^{-}$such that $\sigma(x)=\nu(m)$. Then

$$
\begin{aligned}
\Xi(g) & =\Xi(m)=\Xi\left(m^{-1}\right)=\int_{K} \delta^{1 / 2}\left(h\left(k m^{-1}\right)\right) d k \\
& \geq \delta^{1 / 2}\left(m^{-1}\right) \mu\left(K \cap m^{-1} K m\right)=\delta^{1 / 2}\left(m^{-1}\right) \mu\left(K m^{-1} K\right)^{-1} \\
& =\delta^{1 / 2}\left(m^{-1}\right) \mu(K m K)^{-1} .
\end{aligned}
$$

Now Lemma 1.1 implies the left inequality of (2.1).

Fix $\gamma, 0<\gamma \leq 2$, and fix an open compact subgroup $K_{0}$ of $G$. Set

$$
\boldsymbol{v}_{r}^{\gamma}(f)=\sup \left\{|f(g)| \Xi^{-2 / \gamma}(g)(1+\|\sigma(g)(0)\|)^{r} ; g \in G\right\}
$$

for $r \in \mathbf{R}$ and $f \in C\left(G, K_{0}\right)$. Let

$$
e^{\gamma}\left(G, K_{0}\right)=\left\{f \in C\left(G, K_{0}\right) ; v_{r}^{\gamma}(f)<\infty \text { for all } r \in \mathbf{R}\right\} .
$$

The vector space $e^{\gamma}\left(G, K_{0}\right)$ is topologized by means of the set of semi-norms $v_{r}^{\gamma}, r \in \mathbf{R}$. Then $\mathcal{C}^{\gamma}\left(G, K_{0}\right)$ is a metrizable locally convex space. 
Obviously, $v_{r}^{\gamma_{2}}(f) \leq v_{r}^{\gamma_{1}}(f)$ for $\gamma_{1} \leq \gamma_{2}, r \in \mathbf{R}$, and $f \in C\left(G, K_{0}\right)$. Therefore, $e^{\gamma_{1}}\left(G, K_{0}\right) \subseteq e^{\gamma_{2}}\left(G, K_{0}\right)$. The last inclusion is continuous and dense.

Set

$$
u_{r}^{\gamma}(f)=\sup \left\{|f(g)| \delta^{-1 / \gamma}(\sigma(g))(1+\|\sigma(g)(0)\|)^{r} ; g \in G\right\}
$$

for $r \in \mathbf{R}, f \in C\left(G, K_{0}\right)$. According to Proposition 2.1. we have

$$
e^{\gamma}\left(G, K_{0}\right)=\left\{f \in C\left(G, K_{0}\right) ; u_{r}^{\gamma}(f)<\infty \text { for all } r \in \mathbf{R}\right\} \text {. }
$$

The family of seminorms $u_{r}^{\gamma}, r \in \mathbf{R}$, induces the same topology as the preceding family $v_{r}^{\gamma}, r \in \mathbf{R}$.

2.2. Proposition. The space $e^{\gamma}\left(G, K_{0}\right)$ is a Fréchet space, $C_{0}\left(G, K_{0}\right)$ is a dense subspace of $\mathcal{e}^{\gamma}\left(G, K_{0}\right)$, and the inclusion $\mathcal{C}^{\gamma}\left(G, K_{0}\right) \rightarrow L^{\gamma}\left(G, K_{0}\right)$ is continuous.

Proof. Choose $c>0$ such that $v_{g} \leq c \delta(\sigma(g))^{-1}$ (Lemma 1.1), and $r \in \mathbf{R}$, such that $r \gamma>l$. Then

$$
\int_{G}|f(g)|^{\gamma} d g \leq c\left(u_{r}^{\gamma}(f)\right)^{\gamma} \sum_{t \in T^{-}}(1+\|t(0)\|)^{-r \gamma}
$$

for all $f \in e^{\gamma}\left(G, K_{0}\right)$. Since $\Sigma_{t \in T^{-}}(1+\|t(0)\|)^{-r \gamma}$ is finite, we have $f \in$ $L^{\gamma}\left(G, K_{0}\right)$ and the inclusion is continuous.

The proof of the rest is standard so we omit it.

The next technical lemma plays a crucial role in proving the fact that $\mathrm{e}^{\gamma}\left(G, K_{0}\right)$ is a topological algebra. This result is analogous to the corresponding assertion for real semisimple Lie groups ([7], Ch. 1 Theorem 1).

2.3. Lemma. Choose $r_{1} \in \mathbf{R}$ such that $r_{1}>2 r_{0}+l$. Then the function

$$
g \mapsto \Xi^{2}(g)(1+\|\sigma(g)(0)\|)^{-r_{1}}
$$

is integrable. For $0<\gamma \leq 2$ there exists $c>0$ such that, for $p, q \in \mathbf{R}$,

$$
\begin{gathered}
\int_{G} \Xi^{2 / \gamma}(x) \Xi^{2 / \gamma}\left(x^{-1} y\right)(1+\|\sigma(x)(0)\|)^{-p}\left(1+\left\|\sigma\left(x^{-1} y\right)(0)\right\|\right)^{-q} d x \\
\quad \leq c \Xi^{2 / \gamma}(y)(1+\|\sigma(y)(0)\|)^{-q+(2 / \gamma-1) r_{0}}
\end{gathered}
$$

for all $y \in G$ as soon as

$$
q-p \leq-r_{1}-2 r_{0}(2 / \gamma-1) \text { and } q \geq r_{0}(2 / \gamma-1) .
$$


Proof. Let $c_{1}, c_{2}$ be the real positive numbers from Proposition 2.1. Choose $c_{0}>0$ such that $v_{g} \leq c_{0} \delta(\sigma(g))^{-1}$ (Lemma 1.1). Then

$$
\int_{G} \Xi^{2}(g)(1+\|\sigma(g)(0)\|)^{-r_{1}} d g \leq c_{0} c_{2}^{2} \sum_{t \in T^{-}}(1+\|t(0)\|)^{2 r_{0}-r_{1}} .
$$

As $2 r_{0}-r_{1}<-l$, the series on the right side converges. The integral of the function (2.4) is denoted by $c_{3}$.

From the definition of $\sigma$ and from (1.2) we get

$$
\sigma(x)(0)+\sigma\left(x^{-1} y\right)(0)-\sigma(y)(0) \in-C_{0}^{\perp} .
$$

Since $\sigma(x)(0), \sigma\left(x^{-1} y\right)(0), \sigma(y)(0) \in-\bar{C}_{0}$, we have

$$
\|\sigma(y)(0)\| \leq\left\|\sigma(x)(0)+\sigma\left(x^{-1} y\right)(0)\right\| \leq\|\sigma(x)(0)\|+\left\|\sigma\left(x^{-1} y\right)(0)\right\| .
$$

Thus

$$
(1+\|\sigma(y)(0)\|) \leq(1+\|\sigma(x)(0)\|)\left(1+\left\|\sigma\left(x^{-1} y\right)(0)\right\|\right) .
$$

Relation (1.4) implies $\delta(t) \leq 1$ for all $t \in T_{-}$. We obtain

$$
\delta(\sigma(x)) \delta\left(\sigma\left(x^{-1} y\right)\right) \leq \delta(\sigma(y)) .
$$

From the well-known equality

$$
\int_{K} \Xi(x k y) d k=\Xi(x) \Xi(y)
$$

follows the identity

$$
\begin{aligned}
\int_{G} \Xi(x) \Xi\left(x^{-1} y\right) & (1+\|\sigma(x)(0)\|)^{q-p} d x \\
= & \Xi(y) \int_{G} \Xi^{2}(x)(1+\|\sigma(x)(0)\|)^{q-p} d x .
\end{aligned}
$$

Set $c_{4}=c_{1}^{1-2 / \gamma} c_{2}^{2(2 / \gamma-1)}$. Relations (2.1) and (2.7) imply

$$
\begin{aligned}
& \Xi^{2 / \gamma-1}(x) \Xi^{2 / \gamma-1}\left(x^{-1} y\right) \\
& \quad \leq c_{4} \Xi^{2 / \gamma-1}(y)\left((1+\|\sigma(x)(0)\|)\left(1+\left\|\sigma\left(x^{-1} y\right)(0)\right\|\right)\right)^{r_{0}(2 / \gamma-1)} .
\end{aligned}
$$

Now, from the last inequality, (2.6) and (2.8), we obtain (2.5).

2.4. TheOREM. For $0<\gamma \leq 2$, $\bigodot^{\gamma}\left(G, K_{0}\right)$ is a Fréchet algebra under the convolution. 
Proof. For $r>0$ choose $q, p \in \mathbf{R}$ satisfying $q \geq r+r_{0}(2 / \gamma-1)$ and $q-p \leq-r_{1}-2 r_{0}(2 / \gamma-1)$. The definition of $v_{r}^{\gamma}$ and (2.5) imply

$$
\left|\left(f_{1} * f_{2}\right)(y)\right| \leq c \Xi^{2 / \gamma}(y)(1+\|\sigma(y)(0)\|)^{-q+(2 / \gamma-1) r_{0}} v_{p}^{\gamma}\left(f_{1}\right) v_{q}^{\gamma}\left(f_{2}\right)
$$

for all $f_{1}, f_{2} \in \mathcal{C}^{\gamma}\left(G, K_{0}\right)$ and $y \in G$. Now, it is obvious that $v_{r}^{\gamma}\left(f_{1} * f_{2}\right) \leq$ $c v_{p}^{\gamma}\left(f_{1}\right) v_{q}^{\gamma}\left(f_{2}\right)$.

The proof of the preceding theorem is simpler for $\gamma=2([8]$, Theorem 4.4.2).

Let $K_{0} \subseteq K_{1}$ be open compact subgroups of $G$. Then $\complement^{\gamma}\left(G, K_{1}\right) \subseteq$ $e^{\gamma}\left(G, K_{0}\right)$, the inclusion being continuous and even an isomorphism onto the image. The family $e^{\gamma}\left(G, K_{0}\right)$, with $K_{0}$ an open compact subgroup of $G$, is an inductive system of locally convex spaces. The inductive limit $e^{\gamma}(G)$ of this family is the union of all $\mathcal{C}^{\gamma}\left(G, K_{0}\right)$ over all $K_{0}$. The space $e^{\gamma}(G)$ is a complete locally convex space.

3. Asymptotic behaviour of spherical functions. For $s \in \hat{T}_{\mathbf{C}}$ the mapping $t(0) \mapsto \ln |s(t)|, t \in T$, is a homomorphism from the lattice $\{t(0) ; t \in T\}$ in $\mathbb{Q}$ into the additive group of real numbers. This mapping is uniquely extended to the linear form $s_{1}$ on $\mathbb{Q}$. Set $\eta(s)=\Lambda^{-1}\left(s_{1}\right)$. If $x_{1}, \ldots, x_{l}$ is a basis of $A$ contained in $\{t(0) ; t \in T\}$ and $\tilde{x}_{1}, \ldots, \tilde{x}_{l}$ the basis of $\mathbb{Q}$ biorthogonal to $x_{1}, \ldots, x_{l}$ with respect to the fixed scalar product on $Q$, then

$$
\eta(s)=\sum_{J=1}^{l} \ln \left|s\left(\tau\left(x_{j}\right)\right)\right| \tilde{x}_{j} .
$$

The function $\eta$ is a continuous, open and closed surjection from $\hat{T}_{\mathbf{C}}$ onto $Q$. If $X$ is a compact subset of $Q, \eta^{-1}(X)$ is a compact subset of $\hat{T}_{\mathrm{C}}$. Note that $\eta$ commutes with the action of $W$.

For $s \in \hat{T}_{\mathbf{C}}$, the character $t \mapsto|s(t)|, T \rightarrow \mathbf{R}_{+}^{*}$ is denoted by $|s|$.

3.1. LemMa. (i) For $s \in \hat{T}_{\mathrm{C}}$ and $g \in G$ one has

$$
\left|\Gamma_{s}(g)\right| \leq \Gamma_{|s|}(g) \text {. }
$$

(ii) Let $s \in \hat{T}_{\mathbf{C}}$. If $\eta(s) \in \bar{C}_{0}$, then

$$
\left|\Gamma_{s}\left(m^{-1}\right)\right| \leq \Xi(m)|s|^{-1}(m) \text { for all } m \in M^{-} \text {. }
$$


Proof. (i) is obvious. By the definition of $h$ and (1.1) one has

$$
\nu\left(m^{-1}\right)(0)-h\left(k m^{-1}\right)(0) \in C_{0}^{\perp}
$$

for $k \in K$. Thus $\left|s\left(\mathrm{~m}^{-1}\right)\right| \geq\left|s\left(h\left(\mathrm{~km}^{-1}\right)\right)\right|$. From this inequality we obtain

$$
\begin{aligned}
\left|\Gamma_{s}\left(m^{-1}\right)\right| & \leq \Gamma_{|s|}\left(m^{-1}\right)=\int_{K}|s|\left(h\left(k m^{-1}\right)\right) \delta^{1 / 2}\left(h\left(k m^{-1}\right)\right) d k \\
& \leq|s|^{-1}(m) \Xi(m) .
\end{aligned}
$$

\subsection{Corollary. There exists $c>0$ such that}

$$
\left|\Gamma_{s}\left(m^{-1}\right)\right| \leq c|s|^{-1}(m) \delta^{1 / 2}(m)(1+\|\nu(m)(0)\|)^{r_{0}}
$$

for all $m \in M^{-}$and $s \in \hat{T}_{\mathbf{C}}$ with $\eta(s) \in \bar{C}_{0}$.

The next technical lemma is necessary for a description of the asymptotics of the derivative of spherical functions. This is an analogue of Lemmas 3.3.2.2. and 3.3.2.3. of [12].

3.3. Lemma. Let $w_{0} \in W$ and $w_{0} C_{0}=-C_{0}$. Then one has

$$
\begin{gathered}
\nu(m)(0)-h(k m)(0) \in C_{0}^{\perp}, \\
h(k m)(0)-w_{0}(\nu(m)(0)) \in C_{0}^{\perp}
\end{gathered}
$$

for all $k \in K$ and $m^{-1} \in M^{-}$.

Proof. The first relation is clear. Fix $k \in K, m^{-1} \in M^{-}$and $w \in K$ such that $\nu(w)=w_{0}$. Choose $u_{1} \in U, m_{1} \in M$ and $k_{1} \in K$ such that $\mathrm{km}=u_{1} m_{1} k_{1}$. By definition one has $\nu\left(m_{1}\right)=h(\mathrm{~km})$. Since $\nu\left(w m^{-1} w^{-1}\right)(0)$ $=-w_{0}(\nu(m)(0))$, one now has $\left(w m^{-1} w^{-1}\right)^{-1} \in M^{-}$. (3.5) implies $-w_{0}(\nu(m)(0))-h\left(k_{1} m^{-1}\right)(0) \in C_{0}^{\perp}$. We can find elements $u_{2} \in U$, $m_{2} \in M$ and $k_{2} \in K$ such that $k_{1} m^{-1}=u_{2} m_{2} k_{2}$. Obviously, $k=$ $u_{1}\left(m_{1} u_{2} m_{1}^{-1}\right) m_{1} m_{2} k_{2}$. Since $M$ normalizes $U$, we have $\nu\left(m_{1}\right)=\nu\left(m_{2}\right)^{-1}$. Now (3.6) immediately follows.

If $X \in \mathcal{Q}(\mathrm{t})$ and $g \in G$, then the result of the action of $X$ on the function $s \mapsto \Gamma_{s}(g)$ will be denoted by $\Gamma_{s}(g)^{X}$. Analogously, for $t \in T$, $s(t)^{X}$ will denote the result of the action of $X$ on the function $s \mapsto s(t)$.

In the first section we have fixed a basis $b_{1}, \ldots, b_{l}$ of $T$. Let $\tilde{b}_{1}, \ldots, \tilde{b}_{l}$ be the basis of $A$ biorthogonal to the basis $b_{1}(0), \ldots, b_{l}(0)$. One has

$$
s(t)^{S_{j}}=i\left(\tilde{b}_{j}, t(0)\right) s(t) \quad \text { for } t \in T, j=1, \ldots, l .
$$


For $X \in \mathcal{S}$ there exist $n_{1}, \ldots, n_{l} \in \mathbf{Z}_{+}$such that $X=S_{1}^{n_{1}} \cdots S_{l}^{n_{l}}$. (3.7) implies

$$
s(t)^{X}=s(t) \prod_{J=1}^{l}{ }^{\prime}\left(i\left(b_{J}, t(0)\right)\right)^{n_{J}},
$$

where the product runs over all $j$ with $n_{J} \neq 0$.

3.4. LemMA. For $n \in \mathbf{Z}_{+}$there exists $c>0$ such that

$$
\left|\Gamma_{s}\left(m^{-1}\right)^{X}\right| \leq c(1+\|\nu(m)(0)\|)^{n} \Gamma_{|s|}\left(m^{-1}\right)
$$

for all $X \in \varsigma_{n}$ and $m \in M^{-}$.

Proof. Let $\{\tilde{\alpha} ; \alpha \in \Delta\}$ be the basis of $Q$ biorthogonal to the basis $\{\alpha$; $\alpha \in \Delta\}$. The norm \|\|$_{\infty}$ on $\mathbb{Q}$ is defined by

$$
\|x\|_{\infty}=\max \{|(\tilde{\alpha}, x)| ; x \in \Delta\} .
$$

Choose $w_{0} \in W$ such that $w_{0} C_{0}=-C_{0}$. Relations (3.5) and (3.6) imply $\left\|h\left(\mathrm{~km}^{-1}\right)(0)\right\|_{\infty} \leq\|\nu(m)(0)\|$ for $m \in M^{-}$and $k \in K$. Choose $c_{2} \geq 1$ such that $|(\tilde{b}, x)| \leq c_{2}\|x\|_{\infty}$ for all $x \in \mathbb{Q}, j=1, \ldots, l$. Pick $c_{3} \geq 1$ such that $\|x\|_{\infty} \leq c_{3}\|x\|$ for all $x \in \mathbb{Q}$.

For $X \in \mathcal{S}_{n}$ choose $n_{1}, \ldots, n_{l} \in \mathbf{Z}_{+}$such that $X=S_{1}^{n_{1}} \cdots S_{l}^{n_{l}}$. Then $n_{1}+\cdots+n_{l} \leq n$. From Definitions (1.6) and (3.8) we have

$$
\begin{aligned}
\left|\Gamma_{s}\left(m^{-1}\right)^{X}\right| & \leq \int_{K}\left(\prod_{j=1}^{l}\left|\left(\tilde{b}_{J}, h\left(k m^{-1}\right)(0)\right)\right|^{n j}\right)\left(s \delta^{1 / 2}\right)\left(h\left(k m^{-1}\right)\right) d k \\
& \leq\left(c_{1} c_{2} c_{3}\right)^{n}(1+\|\nu(m)(0)\|)^{n} \Gamma_{|s|}\left(m^{-1}\right) .
\end{aligned}
$$

4. The spherical transformation on $e^{2}(G, K)$. Let $C^{\infty}(\hat{T})$ be the vector space of all infinitely differentiable complex-valued functions on $\hat{T}$. We write

$$
p_{n}(\varphi)=\max \left\{|(X \varphi)(x)| ; X \in \tilde{\mathcal{S}}_{n} \text { and } x \in \hat{T}\right\}
$$

for $\varphi \in C^{\infty}(\hat{T})$ and $n \in \mathbf{Z}_{+}$. The seminorms $p_{n}, n \in \mathbf{Z}_{+}$, topologize $C^{\infty}(\hat{T})$ such that $C^{\infty}(\hat{T})$ is a Fréchet space; moreover, it is a topological algebra under pointwise multiplication. The group $W$ acts on $C^{\infty}(\hat{T})$; let $C_{W}(\hat{T})$ be the subalgebra of all $W$-invariant elements of $C^{\infty}(\hat{T})$. Then $C_{W}^{\infty}(\hat{T})$ is a Fréchet algebra. 
The definition of $\mathcal{C}^{2}(G, K),(2.4)$, and (3.2) imply that the function $g \mapsto f(g) \Gamma_{s}\left(g^{-1}\right)$ is integrable for $s \in \hat{T}$ and $f \in \mathcal{C}^{2}(G, K)$. One now defines $\hat{f}: \hat{T} \rightarrow \mathbf{C}$ by

$$
\hat{f}(s)=\int_{G} f(g) \Gamma_{s}\left(g^{-1}\right) d g .
$$

We call $\hat{f}$ a spherical transform of $f$ and the mapping $f \mapsto \hat{f}$ is called a spherical transformation.

4.1. TheOREM. One has $\hat{f} \in C_{W}^{\infty}(\hat{T})$ for $f \in \mathcal{C}^{2}(G, K)$. The spherical transformation $\hat{e}^{2}(G, K) \rightarrow C_{W}^{\infty}(\hat{T})$ is a morphism of topological algebras with unit.

Proof. Let $f \in \mathcal{C}^{2}(G, K)$. Then (1.8) implies $\hat{f}(s)=\hat{f}(w s)$ for $s \in \hat{T}$ and $w \in W$. Fix $n \in \mathbf{Z}_{+}$and $r_{1}>2 r_{0}+l$. Choose $c>0$ satisfying (3.9). Let $c_{1}$ be the integral of (2.4). One now has

$$
\left|f(g) \Gamma_{s}\left(g^{-1}\right)^{X}\right| \leq c v_{n+r_{1}}^{2}(f)(1+\|\sigma(g)(0)\|)^{-r_{1}} \Xi(g)
$$

for $g \in G$ and $X \in \tilde{\widetilde{\delta}}_{n}$. Since the function on the right side is integrable, we obtain that $f \in C_{W}^{\infty}(\hat{T})$ and

$$
(X \hat{f})(s)=\int_{G} f(g) \Gamma_{s}\left(g^{-1}\right)^{X} d x .
$$

Thus $p_{n}(\hat{f}) \leq c c_{1} v_{n+r_{1}}^{2}(f)$.

It is easy to see that $\left(f_{1} * f_{2}\right)=\hat{f}_{1} \hat{f}_{2}$ for $f_{1}, f_{2} \in \mathcal{C}^{2}(G, K)$.

The cardinal number of $W$ is denoted by $|W|$. For $\varphi \in C_{W}^{\infty}(\hat{T})$ one defines $\varphi: G \rightarrow \mathbf{C}$ by

$$
\varphi \check{\varphi}(g)=\frac{Q}{|W|} \int_{\hat{T}} \varphi(s) \Gamma_{s}(g)|c(s)|^{-2} d s .
$$

where $d s$ is the normalized Haar measure on $\hat{T}$. Clearly, $\varphi \check{\varphi} \in C(G, K)$.

Using Macdonald's formula, we obtain

$$
\check{\varphi}(g)=\delta^{1 / 2}(\sigma(g)) \int_{\hat{T}} \varphi\left(s^{-1}\right) c(s)^{-1} \overline{s(\sigma(g))} d s .
$$

Note that the function $s \mapsto c(s)^{-1}$ belongs to $C^{\infty}(\hat{T})$.

4.2. LemMa. The mapping $\varphi \mapsto \varphi$ from $C_{W}^{\infty}(\hat{T})$ into $C(G, K)$ is injective. 


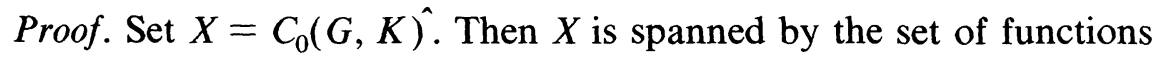
$s \mapsto \Gamma_{s}(g), g \in G$. Using Lemma 1.2. and applying the Stone-Weierstrass Theorem on $C(W \backslash \hat{T})$, we conclude that $X$ is a dense subalgebra of $C_{W}^{\infty}(\hat{T})$ with respect to the supremum norm. One now sees that $\check{\varphi}=0$ implies $\varphi=0$.

For $\varphi \in C^{\infty}(\hat{T})$ and $t \in T$ we write

$$
\varphi_{t}=\int_{\hat{T}} \varphi(s) \overline{s(t)} d s .
$$

Let $\tilde{b}_{1}, \ldots, \tilde{b}_{l}$ be the basis of $Q$ biorthogonal to $b_{1}(0), \ldots, b_{l}(0)$. One has $\left(\tilde{S}_{j} \varphi\right)_{t}=i(\tilde{b}, t(0)) \varphi_{t}$ for $j=1, \ldots, l$. For $X \in \tilde{\delta}$ choose $n_{1}, \ldots, n_{l} \in \mathbf{Z}_{+}$ such that $X=\left(\tilde{S_{1}}\right)^{n_{1}} \cdots\left(\tilde{S}_{l}\right)^{n_{l}}$. We have

$$
(X \varphi)_{t}=\varphi_{t} \prod_{j=1}^{l}\left(i\left(\tilde{b}_{j}, t(0)\right)\right)^{n_{j}} .
$$

4.3. Lemma. To every $n \in \mathbf{Z}_{+}$there corresponds $c_{n}>0$ such that

$$
(1+\|t(0)\|)^{n}\left|\varphi_{t}\right| \leq c_{n} p_{n l}(\varphi) \quad \text { for all } \varphi \in C^{\infty}(\hat{T}) \text { and } t \in T \text {. }
$$

Proof. The norm \|\|$_{\infty}$ on $Q$ is defined by

$$
\|x\|_{\infty}=\max \left\{\left|\left(\tilde{b}_{j}, x\right)\right| ; j=1, \ldots, l\right\} .
$$

Let $c$ be an upper bound of the function $t \mapsto(1+\|t(0)\|)^{n}\|t(0)\|_{\infty}^{-n}$ on the set $T \backslash\{\tau(0)\}$. Set

$$
Y=\left\{j ; 1 \leq j \leq l \text { and }\left(\tilde{b}_{j}, t(0)\right) \neq 0\right\} .
$$

Let $X=\prod_{j \in Y}\left(\tilde{S_{j}}\right)^{n}$. Then (4.7) implies $p_{n l}(\varphi) \geq\|t(0)\|_{\infty}^{n}\left|\varphi_{t}\right|$. One now has $p_{n l}(\varphi) \geq c^{-1}(1+\|t(0)\|)^{n}\left|\varphi_{t}\right|$ for $t \neq \tau(0)$.

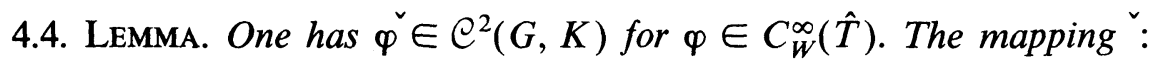
$C_{W}^{\infty}(\hat{T}) \rightarrow \mathcal{C}^{2}(G, K)$ is a monomorphism of topological vector spaces.

Proof. For $n \in \mathbf{Z}_{+}$let $c_{n}$ satisfy (4.8). Then (4.5) and (4.8) imply $u_{n}^{2}(\varphi) \leq c_{n} p_{n l}\left(\varphi\left(s^{-1}\right) c(s)^{-1}\right)$ for $\varphi \in C_{W}^{\infty}(\hat{T})$. Clearly, there exists $c>0$ such that for all $\varphi \in C_{W}^{\infty}(\hat{T})$ the following inequality holds true:

$$
u_{n}^{2}(\varphi) \leq c p_{n l}\left(\varphi\left(s^{-1}\right) c(s)^{-1}\right) \text {. }
$$

In the rest of the section we shall assume $K$ is a standard maximal compact subgroup. The next lemma is a part of the proof of Theorem (5.1.2) of [5] and here we omit the proof. 
4.5. LemMa. For $m_{1}, m_{2} \in M^{-}$the integral

$$
\int_{\hat{T}} \Gamma_{s}\left(m_{1}\right) \overline{\Gamma_{s}\left(m_{2}\right)}|c(s)|^{-2} d s
$$

is equal to $|W| Q^{-1} v_{m_{1}}^{-1}$ when $\nu\left(m_{1}\right)=\nu\left(m_{2}\right)$. Otherwise this integral is zero.

The immediate consequence of the previous lemma is the fact that $Q|W|^{-1}|c(s)|^{-2} d s$ is the Plancherel measure for $G / K$ on $\hat{T}$.

4.6. Corollary. One has $(\hat{f})^{\nu}=$ f for all $f \in \mathcal{C}^{2}(G, K)$.

From the results of the previous lemmas we easily obtain the next theorem.

4.7. TheOREM. Let $K$ be a standard maximal compact subgroup of $G$. The spherical transformation

$$
\hat{:} \mathfrak{C}^{2}(G, K) \rightarrow C_{W}^{\infty}(\hat{T}), \quad \hat{f}(s)=\int_{G} f(g) \Gamma_{s}\left(g^{-1}\right) d g
$$

is an isomorphism of topological algebras. The inverse formula is given by

$$
f(g)=\frac{Q}{|W|} \int_{\hat{T}} \hat{f}(s) \Gamma_{s}(g)|c(s)|^{-2} d s .
$$

5. The spherical transformation on $\mathrm{e}^{\gamma}(G, K), \gamma<2$. Fix $\gamma, 0<\gamma$ $<2$, for the whole section. We write

$$
D(x)=\bigcap_{w \in W} w\left(x-C_{0}^{\perp}\right)
$$

for $x \in \bar{C}_{0}$. It is a closed, convex, $W$-invariant subset of $\mathscr{Q}$. One has

$$
D(x) \cap \bar{C}_{0}=\left(x-C_{0}^{\perp}\right) \cap \bar{C}_{0} .
$$

Thus, $D(x)$ is a compact subset of $Q$.

By (1.4) one sees that $\eta\left(\delta^{1 / \gamma-1 / 2}\right)=(1 / \gamma-1 / 2) \eta(\delta) \in C_{0}$. The set $D\left(\eta\left(\delta^{1 / \gamma-1 / 2}\right)\right)$ is denoted by $D_{\gamma}$. The interior of $D_{\gamma}$ contains 0 .

The interior of $\eta^{-1}\left(D_{\gamma}\right)$ is denoted by $D^{\gamma}$. It is an open, $W$-invariant set with compact closure and contains $\hat{T}$.

For $\varphi$ an analytic function on $D^{\gamma}$ and $n \in \mathbf{Z}_{+}$set

$$
p_{n}^{\gamma}(\varphi)=\sup \left\{|(X \varphi)(x)| ; X \in \mathcal{S}_{n} \text { and } x \in D^{\gamma}\right\} .
$$


The vector space of all analytic functions $\varphi$ on $D^{\gamma}$ such that $p_{n}^{\gamma}(\varphi)<\infty$ for all $n \in \mathbf{Z}_{+}$is denoted by $\mathcal{H}\left(D^{\gamma}\right)$. The seminorms $p_{n}^{\gamma}, n \in \mathbf{Z}_{+}$, generate the structure of the Fréchet space on $\mathcal{H}\left(D^{\gamma}\right)$, and $\mathcal{H}\left(D^{\gamma}\right)$ is a topological algebra under pointwise multiplication. Denote by $\mathcal{H}_{W}\left(D^{\gamma}\right)$ the subalgebra of all $W$-invariant functions in $\mathcal{H}\left(D^{\gamma}\right)$. It is a Fréchet algebra.

Let $f \in \mathcal{C}^{\gamma}(G, K)$ and $s \in D^{\gamma}$. We will show that the function $g \mapsto$ $f(g) \Gamma_{s}\left(g^{-1}\right)$ is integrable. Choose $w \in W$ such that $w \eta(s) \in \bar{C}_{0}$. It is easy to see that $\left(\delta^{1 / \gamma-1 / 2}|w s|^{-1}\right)(m) \leq 1$ for all $m \in M^{-}$. By Corollary 3.2 there exists $c_{1}>0$ such that

$$
\left|\Gamma_{s}\left(m^{-1}\right)\right| \leq c_{1} \delta^{1-1 / \gamma}(m)(1+\|\nu(m)(0)\|)^{r_{0}}
$$

for all $m \in M^{-}$. Choose $r \in \mathbf{Z}_{+}$such that $r-r_{0}>2 r_{0}+l$. Then there exists $c_{2}>0$ such that

$$
\left|f(m) \Gamma_{s}\left(m^{-1}\right)\right| \leq c_{2} u_{r}^{\gamma}(f) \Xi^{2}(m)(1+\|\nu(m)(0)\|)^{r_{0}-r}
$$

for all $m \in M^{-}$. By (2.4), the right side is integrable, so is the left side.

For $f \in \mathcal{C}^{\gamma}(G, K)$ we define the function $\hat{f}: D^{\gamma} \rightarrow \mathbf{C}$ by

$$
\hat{f}(s)=\int_{G} f(g) \Gamma_{s}\left(g^{-1}\right) d g .
$$

5.1. Theorem. One has $\hat{f} \in \mathcal{H}_{W}\left(D^{\gamma}\right)$ for $f \in \mathcal{C}^{\gamma}(G, K)$. The spherical transformation $\hat{:} \mathcal{C}^{\gamma}(G, K) \rightarrow \mathcal{H}_{W}\left(D^{\gamma}\right)$ is a morphism of topological algebras with unit.

Proof. Let $f \in \mathcal{C}^{\gamma}(G, K), s \in D^{\gamma}$ and $n \in \mathbf{Z}_{+}$. Formulas (3.9) and (5.3) imply

$$
\left|f(m) \Gamma_{s}\left(m^{-1}\right)^{X}\right| \leq c c_{2}(1+\|\nu(m)(0)\|)^{n+r_{0}-r} \Xi^{2}(m) u_{r}^{\gamma}(f)
$$

for $r-n-r_{0}>2 r_{0}+l$. Set $r_{1}=r-n-r_{0}$, and the integral (2.4) is denoted by $c_{3}$. Since the function $g \mapsto f(g) \Gamma_{s}\left(g^{-1}\right)^{X}$ is integrable, we have $\hat{f} \in \mathcal{H}_{W}\left(D^{\gamma}\right)$ and

$$
(X \hat{f})(s)=\int_{G} f(g) \Gamma_{s}\left(g^{-1}\right)^{X} d g .
$$

(5.4) implies $p_{n}^{\gamma}(\hat{f}) \leq c c_{2} c_{3} u_{r}^{\gamma}(f)$.

In the rest of this section we assume $K$ is a standard maximal compact subgroup.

For $\varphi \in \mathcal{H}_{W}\left(D^{\gamma}\right),(\varphi \mid \hat{T})^{\nu}$ is again denoted by $\varphi^{\nu}$. 


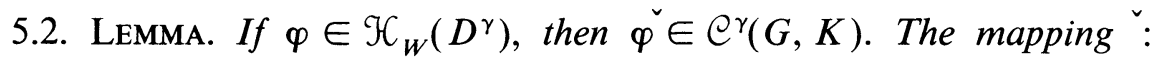
$\mathcal{H}_{W}\left(D^{\gamma}\right) \rightarrow \mathcal{C}^{\gamma}(G, K)$ is a monomorphism of topological vector spaces.

Proof. The proof proceeds in several parts.

(1) Let $Y_{1}$ denote the set of zeros of the function $c(s)$. Since $K$ is standard, one has $\eta\left(Y_{1}\right) \cap\left(-\bar{C}_{0}\right)=\varnothing . \eta\left(Y_{1}\right)$ is a closed subset of $Q$. There exists an open subset $Y_{2}$ of $\mathbb{Q}$ containing $\bar{C}_{0}$ such that the closure of $Y_{2}$ is adjoint with $\left(-\eta\left(Y_{1}\right)\right)$. Set $Y=\eta^{-1}\left(Y_{2}\right) \cap D^{\gamma}$. Then, for $X \in \mathcal{Q}(\mathrm{t})$, $X\left(c^{-1}\left(s^{-1}\right)\right)$ is a bounded function on $Y$.

(2) The unit circle in $\mathbf{C}$ is denoted by $\Gamma$. One now has

$$
\int_{\hat{T}} \psi(s) d s=(2 \pi i)^{-l} \int_{\Gamma} \cdots \int_{\Gamma} \psi\left(\Theta^{-1}\left(z_{1}, \ldots, z_{l}\right)\right) \frac{d z_{1}}{z_{1}} \cdots \frac{d z_{l}}{z_{l}}
$$

for $\psi \in C(\hat{T})$.

For an analytic function $\varphi$ on $Y$ and $n \in \mathbf{Z}_{+}$we write

$$
\|\varphi\|_{n}=\sup \left\{|(X \varphi)(s)| ; X \in \mathcal{S}_{n} \text { and } s \in Y\right\} .
$$

For $t \in T$ we set $(\varphi \mid \hat{T})_{t}=\varphi_{t}$.

We first claim that

$$
\left|\varphi_{t}\right| \delta(t)^{1 / \gamma-1 / 2} \leq\|\varphi\|_{0}
$$

for any analytic function $\varphi$ on $Y$ and $t \in T$.

If $\gamma<\gamma_{1}<2$, then $\delta^{1 / \gamma_{1}-1 / 2} \in Y$. Let $\tilde{b}_{1}, \ldots, \tilde{b}_{l}$ be the basis of $A$ biorthogonal to the basis $b_{1}(0), \ldots, b_{l}(0)$. Then

$$
\eta(s)=\sum_{j=1}^{l} \ln \left|s\left(b_{j}\right)\right| \tilde{b}_{j} \quad \text { for } s \in \hat{T}_{\mathbf{C}} .
$$

Since the line connecting 0 and $\eta\left(\delta^{1 / \gamma_{1}-1 / 2}\right)$ is contained in $Y_{2}$, and $Y_{2}$ is open, we can find points $y_{0}, \ldots, y_{m} \in Y_{2}$ such that $y_{0}=0, y_{m}=$ $\eta\left(\delta^{1 / \gamma_{1}-1 / 2}\right)$ and such that the vector $y_{j}-y_{j-1}$ is collinear with an element of $\left\{\tilde{b}_{1}, \ldots, \tilde{b}_{l}\right\}$ for every $j, 1 \leq j \leq m$. Choose $r_{j} \in \hat{T}_{\mathbf{C}}$ such that $\eta\left(r_{j}\right)=y_{j}$ and $r_{j}=\left|r_{j}\right|, j=0, \ldots, m$. The positively oriented circle in $C$ with center 0 and radius $r_{n}\left(b_{j}\right)$ is denoted by $\Gamma_{j}^{n}$.

For $t \in T$ choose $n_{1}, \ldots, n_{l} \in \mathbf{Z}$ such that $t=b_{1}^{n_{1}} \cdots b_{l}^{n_{l}}$. Then one has

$$
\varphi_{t}=(2 \pi i)^{-l} \int_{\Gamma_{1}^{n}} \cdots \int_{\Gamma_{l}^{n}} \varphi\left(\Theta^{-1}\left(z_{1}, \ldots, z_{l}\right)\right) \frac{d z_{1}}{z_{1}^{1+n_{1}}} \cdots \frac{d z_{l}}{z_{l}^{1+n_{l}}}
$$

for $n=0$. Applying Cauchy's Theorem, we find that (5.8) is valid for $0 \leq n \leq m$. Now (5.8) with $n=m$ implies $\left|\varphi_{t}\right| \leq\|\varphi\|_{0} \delta^{1 / 2-1 / \gamma_{1}}(t)$ for all $\gamma_{1}, \gamma<\gamma_{1}<2$. Thus, we have proved (5.7). 
(3) Fix $r \in \mathbf{Z}_{+}$and let $\varphi$ be an analytic function on $Y$ such that $X \varphi$ is a bounded function on $Y$ for every $X \in \mathcal{S}_{l r}$. Let \|\|$_{\infty}$ be the norm on $\mathbb{Q}$ defined by $\|x\|_{\infty}=\max \left\{\left|\left(\tilde{b}_{n}, x\right)\right| ; 1 \leq n \leq l\right\}$. Let $c_{1}>1$ be an upper bound of the function $t \mapsto(1+\|t(0)\|)^{r}\|t(0)\|_{\infty}^{-r}$ on $T \backslash\{\tau(0)\}$.

For $t \in T$ set $X=\left\{n ; 1 \leq n \leq l\right.$ and $\left.\left(\tilde{b}_{n}, t(0)\right) \neq 0\right\}$. One now has

$$
\left|\left(\left(\prod_{x \in X} S_{n}^{r}\right) \varphi\right)_{t} \geq\|t(0)\|_{\infty}^{r}\right| \varphi_{t} \mid .
$$

Using (5.7), we obtain

$$
(1+\|t(0)\|)^{r}\left|\varphi_{t}\right| \leq c_{1}\|\varphi\|_{l r} \delta(t)^{1 / 2-1 / \gamma} .
$$

(4) For $r \in \mathbf{Z}_{+}$it is easy to see there exists $c_{2}>0$ such that

$$
\left\|\varphi(s) c\left(s^{-1}\right)^{-1}\right\|_{l r} \leq c_{2} p_{r l}^{\gamma}(\varphi) \text { for all } \varphi \in \mathcal{H}\left(D^{\gamma}\right) .
$$

By what we have shown we obtain

$$
u_{r}^{\gamma}(\varphi) \leq c_{1} c_{2} p_{r l}^{\gamma}(\varphi) \quad \text { for all } \varphi \in \mathcal{H}_{W}\left(D^{\gamma}\right)
$$

Thus, $\varphi \check{\in} \in \mathcal{C}^{\gamma}(G, K)$, and the mapping $\varphi \mapsto \varphi^{\nu}$ is continuous.

(5) According to Lemma 4.2, to prove that $\varphi \mapsto \varphi$ is injective, it is sufficient to show that $\varphi \mid \hat{T}=0$ implies $\varphi=0$. This is an immediate consequence of (1.5).

Now, the next theorem is proved.

5.3. THEOREM. Let $K$ be a standard maximal compact subgroup of $G$. The spherical transformation

$$
\hat{:} \mathcal{C}^{\gamma}(G, K) \rightarrow \mathcal{H}_{W}\left(D^{\gamma}\right), \quad \hat{f}(s)=\int_{G} f(g) \Gamma_{s}\left(g^{-1}\right) d g
$$

is an isomorphism of topological algebras. The inversion formula is given by

$$
f(g)=\frac{Q}{|W|} \int_{\hat{T}} \hat{f}(s) \Gamma_{s}(g)|c(s)|^{-2} d s
$$

for all $f \in \mathcal{C}^{\gamma}(G, K)$.

6. The exception case. In this section we shall state without proofs the results about spherical transformations on $\mathcal{C}^{2}(G, K)$ with $K$ an exceptional maximal compact subgroup. We restrict ourselves to $G$, the group of rational points of a simply connected almost simple algebraic group defined over $k$. We assume $K$ is exceptional. 
6.1. THEOREM. Set

$$
\begin{aligned}
& I=\left\{f \in \mathcal{C}^{2}(G, K) ; \int_{G} f(g) \Gamma_{s}\left(g^{-1}\right) d g=0 \text { for all } s \in \hat{T}\right\}, \\
& J=\left\{f \in \mathcal{C}^{2}(G, K) ; f * g=0 \text { for all } g \in I\right\} .
\end{aligned}
$$

Then $I$ and $J$ are closed two-sided ideals in $\mathrm{e}^{2}(G, K)$ and

$$
e^{2}(G, K)=I \oplus J
$$

The spherical transformation

$$
\hat{:} \mathcal{C}^{2}(G, K) \rightarrow C_{W}^{\infty}(\hat{T}), \quad \hat{f}(s)=\int_{G} f(g) \Gamma_{s}\left(g^{-1}\right) d g
$$

is an epimorphism of topological algebras. The kernel of this epimorphism is I. The restriction of the spherical transformation (6.1) to $J$ is an isomorphism

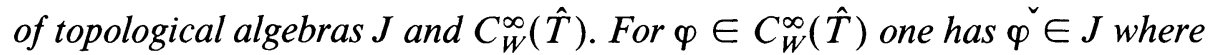

$$
\check{\varphi}(g)=\frac{Q}{|W|} \int_{\hat{T}} \varphi(s) \Gamma_{s}(g)|c(s)|^{-2} d s .
$$

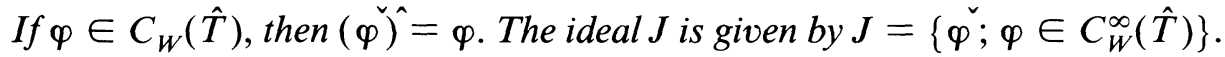
One has $I \neq\{0\}$ for $K$ exceptional.

6.2. Note. Suppose that $G$ is a rank one group, i.e. $l=1$. Then it can be shown that spherical transformation ${ }^{\wedge} \mathcal{C}^{\gamma}(G, K) \rightarrow \mathcal{H}_{W}\left(D^{\gamma}\right), 0<\gamma<$ 2 , is an epimorphism of topological algebras and a statement analogous to Theorem 6.1 is true.

6.3. Note. We can determine $0<\gamma_{1}<2$ depending on $q_{\alpha}, \alpha \in \Sigma_{1}$, such that the assumption in Lemma 5.2 can be replaced by the assumption that $\gamma_{1}<\gamma<2$, and the statement and proof of Lemma are valid with this assumption.

Now, if $\gamma_{1}<\gamma<2$ the spherical transformation is an epimorphism of the topological algebra $\mathcal{C}^{\gamma}(G, K)$ onto $\mathcal{K}_{W}\left(D^{\gamma}\right)$ and the analogue of Theorem 6.1 is true.

\section{REFERENCES}

[1] F. Bruhat and J. Tits, Groupes réductifs sur un corps local, Chapter I: Données radicielles valuées, Publ. Math. I.H.E.S., 41 (1972), 5-251.

[2] W. Casselman, Introduction to the theory of admissible representations of $\mathfrak{p}$-adic reductive groups, preprint.

[3] - The unramified principal series of $\mathfrak{p}$-adic groups I. The spherical function, Comp. Math., 40 (1980), 387-406. 
[4] Harish-Chandra, Harmonic analysis on reductive $\mathfrak{p}$-adic groups, Proc. Sympos. Pure Math. XXVI, Amer. Math. Soc., Providence, 1973, 167-192.

[5] I. G. Macdonald, Spherical functions on a group of p-adic type, Ramanujan Institute, Univ. of Madras Publ., 1971.

[6] _ Spherical functions on a p-adic Chevalley groups, Bull. Amer. Math. Soc., 74 (1968), 520-525.

[7] D. Miličic, Asymptotic behaviour of matrix coefficients of the discrete series, Duke Math. J., 44 (1977), 59-88.

[8] A. J. Silberger, Introduction to Harmonic Analysis on Reductive p-adic Groups, Princeton University Press, Princeton, New Jersey, 1979.

[9] R. Steinberg, Lectures on Chevalley Groups, Yale University, 1967.

[10] J. Tits, Reductive groups over local fields, Proc. Symp. Pure Math. XXXIII, Amer. Math. Soc., Providence, 1978.

[11] P. C. Trombi and V. S. Varadarajan, Spherical transforms on semisimple Lie groups, Ann. Math., 94 (1971), 246-303.

[12] G. Warner, Harmonic Analysis on Semi-Simple Lie Groups I, II, Springer-Verlag, Berlin, 1972.

Received October 10, 1981. The author was supported by SIZ VI SRH.

UNIVERSITY OF ZAGREB

P.O. Box 187

41000 ZaGReB, Yugoslavia 



\section{PACIFIC JOURNAL OF MATHEMATICS}

EDITORS

Donald BABBITt (Managing Editor)

University of California

Los Angeles, CA 90024

Hugo Rossi

University of Utah

Salt Lake City, UT 84112

C. C. Moore and Arthur Ogus

University of California

Berkeley, CA 94720

\section{J. DugundiI}

Department of Mathematics

University of Southern California

Los Angeles, CA 90089-1113

R. Finn and H. SAMELSON

Stanford University

Stanford, CA 94305

\section{ASSOCIATE EDITORS}
R. ARENS
E. F. BECKENBACH
B. H. NEUMANN
F. WOLF
K. YOSHIDA (1906-1982)

\section{SUPPORTING INSTITUTIONS}

UNIVERSITY OF ARIZONA

UNIVERSITY OF BRITISH COLUMBIA

CALIFORNIA INSTITUTE OF TECHNOLOGY

UNIVERSITY OF CALIFORNIA

MONTANA STATE UNIVERSITY

UNIVERSITY OF NEVADA. RENO

NEW MEXICO STATE UNIVERSITY

OREGON STATE UNIVERSITY
UNIVERSITY OF OREGION

UNIVERSITY OF SOUTHERN CALIFORNIA

STANFORD UNIVERSITY

UNIVERSITY OF HAWAII

UNIVERSITY OF TOKYO

UNIVERSITY OF UTAH

WASHINGTON STATE UNIVERSITY

UNIVERSITY OF WASHINGTON 


\section{Pacific Journal of Mathematics}

Vol. 109, No. $1 \quad$ May, 1983

Donald George Babbitt and V. S. Varadarajan, Formal reduction theory of meromorphic differential equations: a group theoretic view $\ldots \ldots \ldots \ldots 1$

Jo-Ann Deborah Cohen, Norms on $F(X) \ldots \ldots \ldots \ldots \ldots \ldots \ldots \ldots \ldots \ldots$

Robert Fitzgerald, Witt kernels of function field extensions $\ldots \ldots \ldots \ldots \ldots 89$

Hervé Jacquet and Joseph Andrew Shalika, The Whittaker models of induced representations .............................. 107

Masakiti Kinukawa, Some generalizations of contraction theorems for

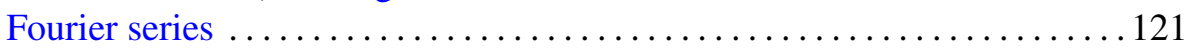

Joseph Weston Kitchen, Jr. and David A. Robbins, Sectional

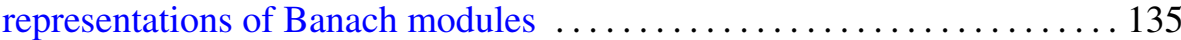

Victor Charles Pestien, Jr., Weak approximation of strategies in measurable gambling

Richard Scott Pierce and Charles Irvin Vinsonhaler, Realizing central

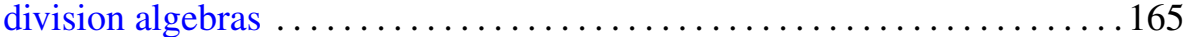

Walter Ricardo Ferrer Santos, Cohomology of comodules ............ 179

Marko Tadić, Harmonic analysis of spherical functions on reductive groups

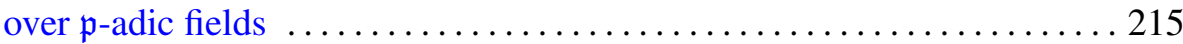

Lorenzo Traldi, The determinantal ideals of link modules. II . . . . . . . . 237

Alain J. Valette, A remark on the Kasparov groups $\operatorname{Ext}^{i}(A, B) \ldots \ldots \ldots 247$ 